related to one particular problem. The book's strength lies in its breadth and generality, and I recommend it without hesitation.

Lancaster University

AN Letchford

\section{Decision Making: Recent Developments and Worldwide Applications}

SH Zanakis, G Doukidis and C Zopounidis (Eds)

Kluwer Academic Publishers, 2000. xv +489 pp. $£ 125.00$ ISBN: 0-7923-6621-2

This book is a collection of papers derived from the Decision Sciences Institute International Conference held in Athens in July 1999. I attended and, in fact, am a co-author of one of the papers in this collection. It was a good conference, but one with many parallel sessions crammed with papers: so many that the 2000-page proceedings distributed to participants limited each paper to an extended abstract of at most four sides. It was impossible, with so many papers, to attend all those of interest and very difficult to find the authors to garner further details. This collection is an attempt to provide, in one edited volume, a cross section of the papers, focusing on decision making and methodologies for its support in a wide range of application areas.

There are 30 papers in this volume divided into the nine areas of: management information systems; education innovations and distance learning; international business; marketing; finance and banking; optimisation and decision making; multi-criteria decision analysis, aid and practice; decision support systems and information technology and; health care planning and hospital operations.

As is immediately obvious, the volume cuts across many disciplines from operational research through decision support systems to management and business. I enjoyed reading it and found the depth in each paper that had been lacking in the four-page abstracts distributed at the conference. I believe that readers of JORS will find much of value here too.

So would I recommend buying this edited collection? Well...

Over the years in several reviews I have remarked on Kluwer's pricing: they have clearly decided to market to libraries and ignore the possibility of sales to any individual of less than millionaire status. But a price of $£ 125$ for a 500page text produced from camera ready copy reaches new heights of absurdity. However good the contents, it is still a conference proceedings. Libraries would be much better advised to spend their money on textbooks, monographs and journals. As a contributor to this volume, I feel that I and the authors of all the papers have put considerable effort into presenting (and formatting!) our work only to have them condemned to obscurity by the policies of Kluwer. Quite simply, this book is not worth buying at this price. If you want to follow up the topics covered in this volume, you will find many equivalent papers in JORS and other journalseven in more modestly priced conference proceedings.

Manchester Business School

S French

\section{Energy Decisions and the Environment: A Guide to the Use of Multicriteria Methods}

BF Hobbs and P Meier

Kluwer Academic Publishers, 2000. vii +257 pp. $£ 70.00$ ISBN: 0-7923-7875-X

This is a nicely written text discussing the application of some of the methodologies in the field of multiple criteria decision analysis (MCDA) to the energy industry. The acronym MCDM (multi-criteria decision-making) is used throughout to describe the methods, but multi-objective programming techniques are not in essence considered. For example, goal programming is discussed, but mainly alongside multiattribute utility theory and outranking techniques as an 'amalgamation method'. This book does not provide an in-depth examination of the theory behind the methods discussed, and is not intended to, but concentrates on the requirements for potential application in a particular framework of analysis.

The introduction acquaints the reader with the type of questions that may be asked in an energy managementbased context, and how multi-criteria methods may be able to help. There is also a useful and well-balanced section outlining reasons why it may not be best to monetize all criteria when including environmental factors into the decision-making process.

The remaining structure of the book is very good and can be split into four key sections. Chapters 2-5 discuss the methods themselves and their application. This is done in 10 steps: steps 1-3 consider the application of the methods detailing attribute selection and definition, option definitions and impact quantification; steps 4-6 detail screening and tradeoff analysis; steps 7-8 deal with value scaling, weighting methods and amalgamation methods; and step 10 looks at the final stage of the resolution of differences amongst methods and individuals. Chapter 6 offers an illustrative example describing a hypothetical energy supply problem in a rural area of a developing country. Specific reference to steps 4-9 is taken for the example, which includes four alternative plans with three attributes. Chapter 7 is a review of MCDM applications in the field of energy planning and policy development, selecting nine specific applications for discussion and analysis. Finally, Chapters 8-10 detail three separate studies that the authors have undertaken in this area: namely at British Columbia (BC) Hydro, BC Gas and Seattle City Light.

A criticism of the book could be that a global view of MCDM is not given. However, as mentioned previously, this 